\title{
Integrated Architecture for Aircraft Engine Performance Monitoring and Fault Diagnostics: Engine Test Results
}

\author{
Aidan W. Rinehart \\ Vantage Partners LLC \\ 3000 Aerospace Parkway \\ Brook Park, OH 44142
}

\author{
Donald L. Simon \\ NASA Glenn Research Center \\ 21000 Brookpark Road \\ Cleveland, $\mathrm{OH} 44135$
}

AIAA Joint Propulsion Conference 2014

July 28-30, 2014

Cleveland, $\mathrm{OH}$ 


\section{Overview}

- Background

- Architecture

- Application

- Results

- Conclusion 


\section{Background - aircraft engine performance trend monitoring and gas path fault diagnostics}

- Conventional Approach:

- Ground-based

- Processing of "snapshot" measurements post-flight

- Enables estimation and trending of engine performance and gas path fault diagnostics

- Early diagnosis of incipient fault conditions with minimal latency can be challenging

- Emerging Approach:

- Advances in on-board processing and flight data recording capabilities are enabling new diagnostic approaches

- Acquisition of full-flight streaming/continuous measurement data now possible

- Requires new approaches to analyze expanded quantity and format of data
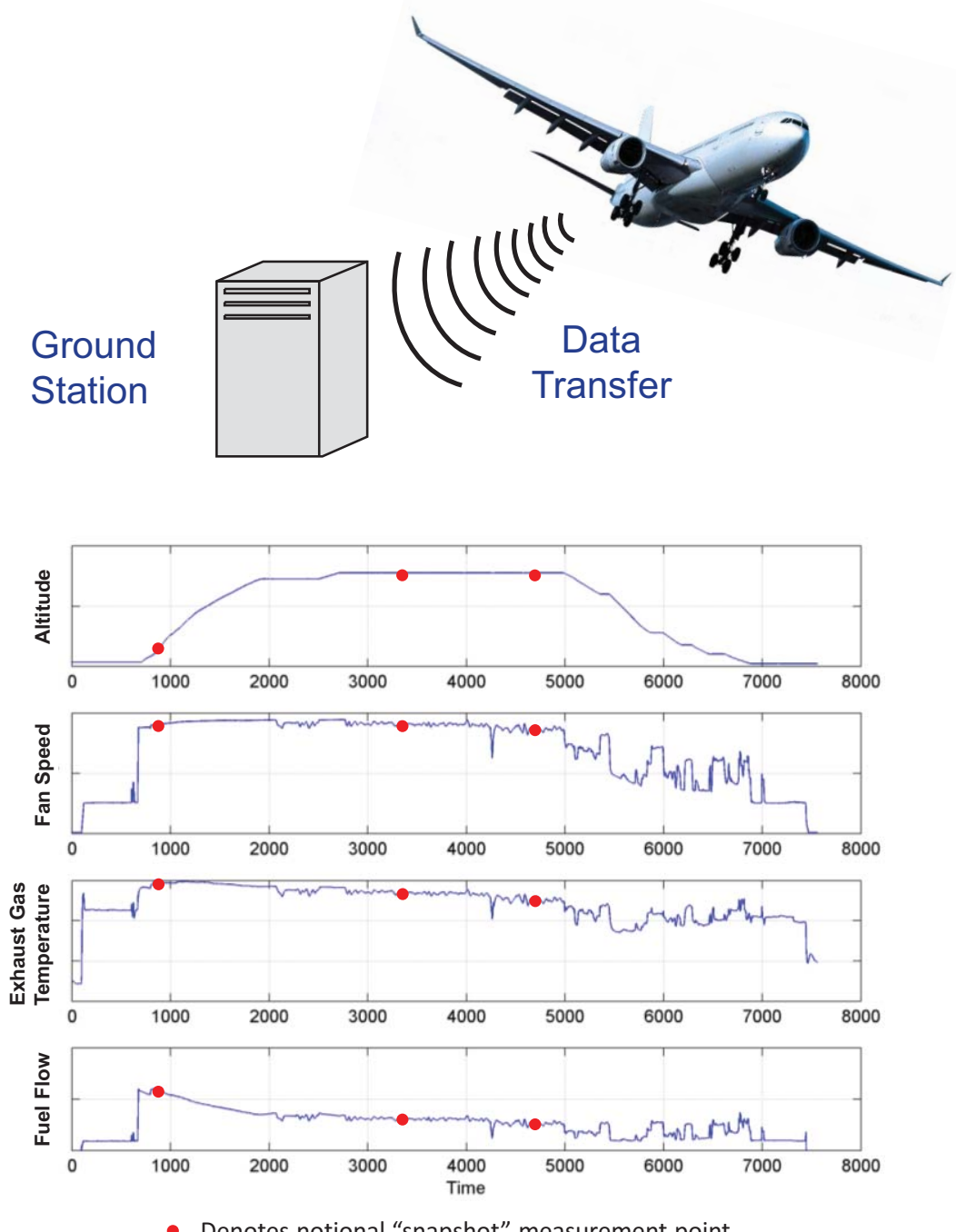

- Denotes notional "snapshot" measurement point

Example Aircraft Engine Flight Data 


\section{Architecture for Engine Performance Monitoring and Fault Diagnostics}

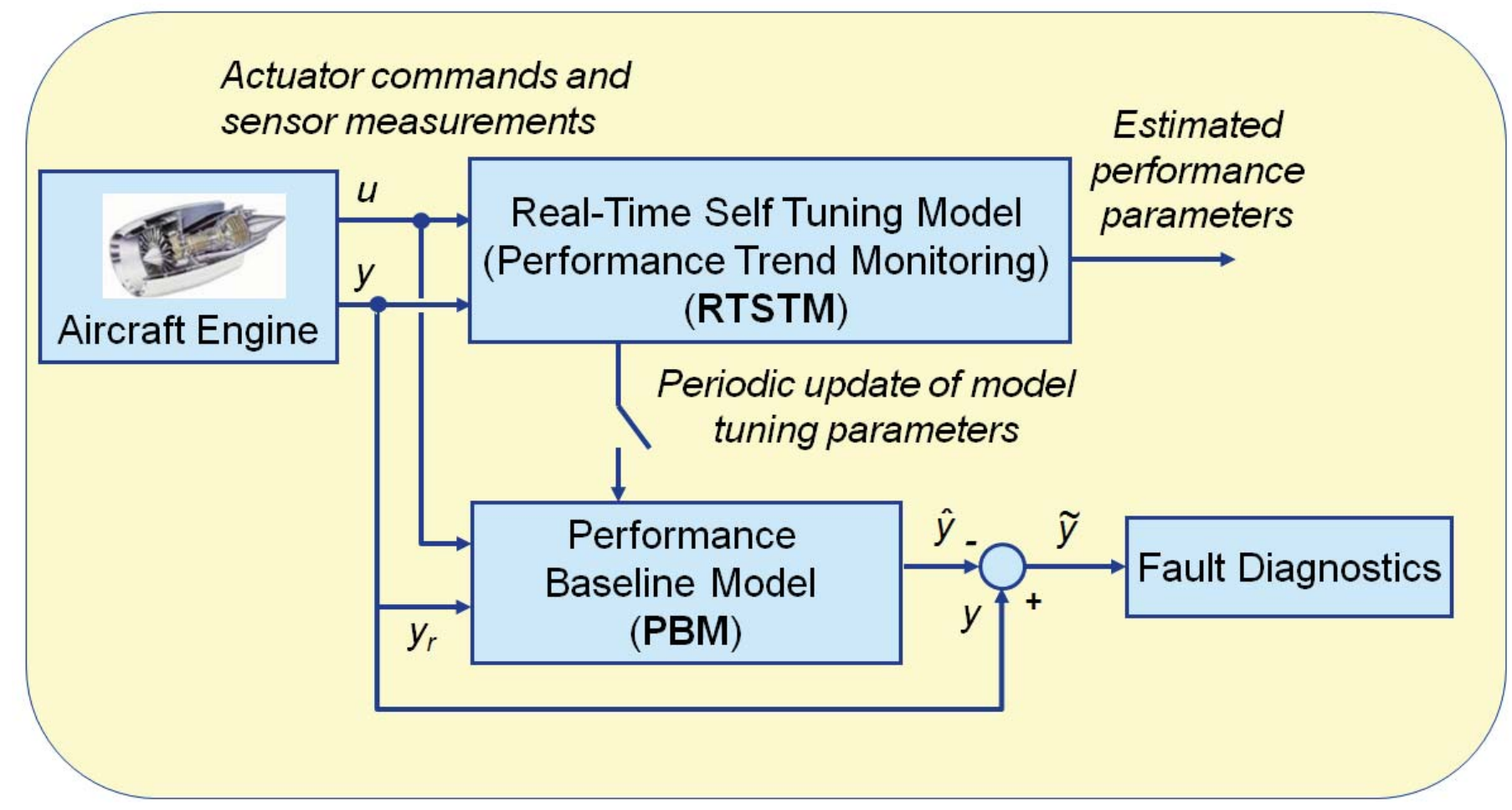

- Designed for processing real-time continuous (streaming) engine measurement data to provide:

- Estimation and trending of deterioration-induced engine performance changes

- Detection and isolation of gas path system faults 


\section{Real-Time Self Tuning Model}

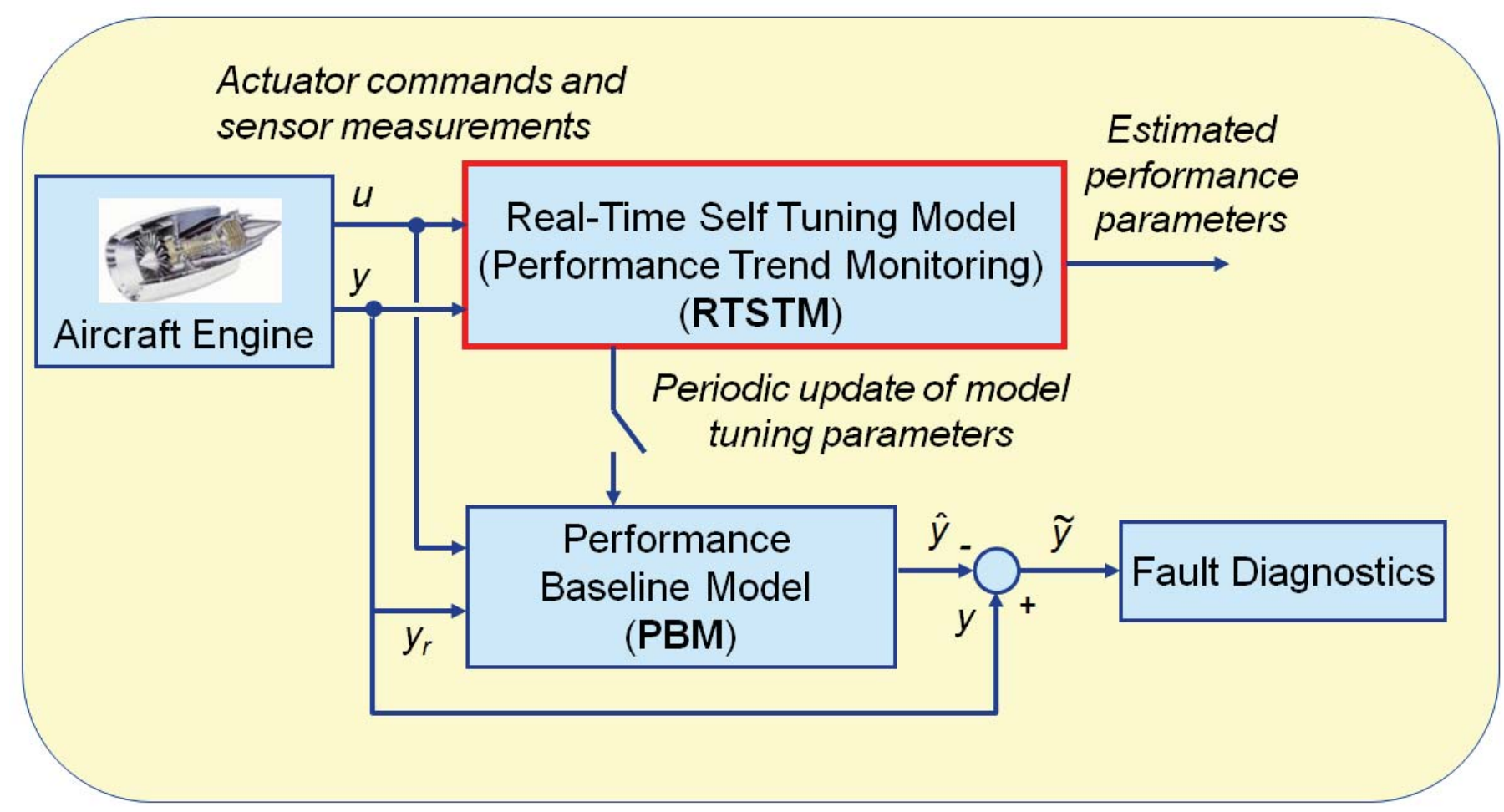

- Self-tuning piecewise linear Kalman filter design

- Applies NASA-developed optimal tuner selection

- Application for underdetermined estimation problems

- Minimizes mean squared estimation error in parameters of interest

- Provides real-time estimates of unmeasured engine performance parameters 


\section{Performance Baseline Model}

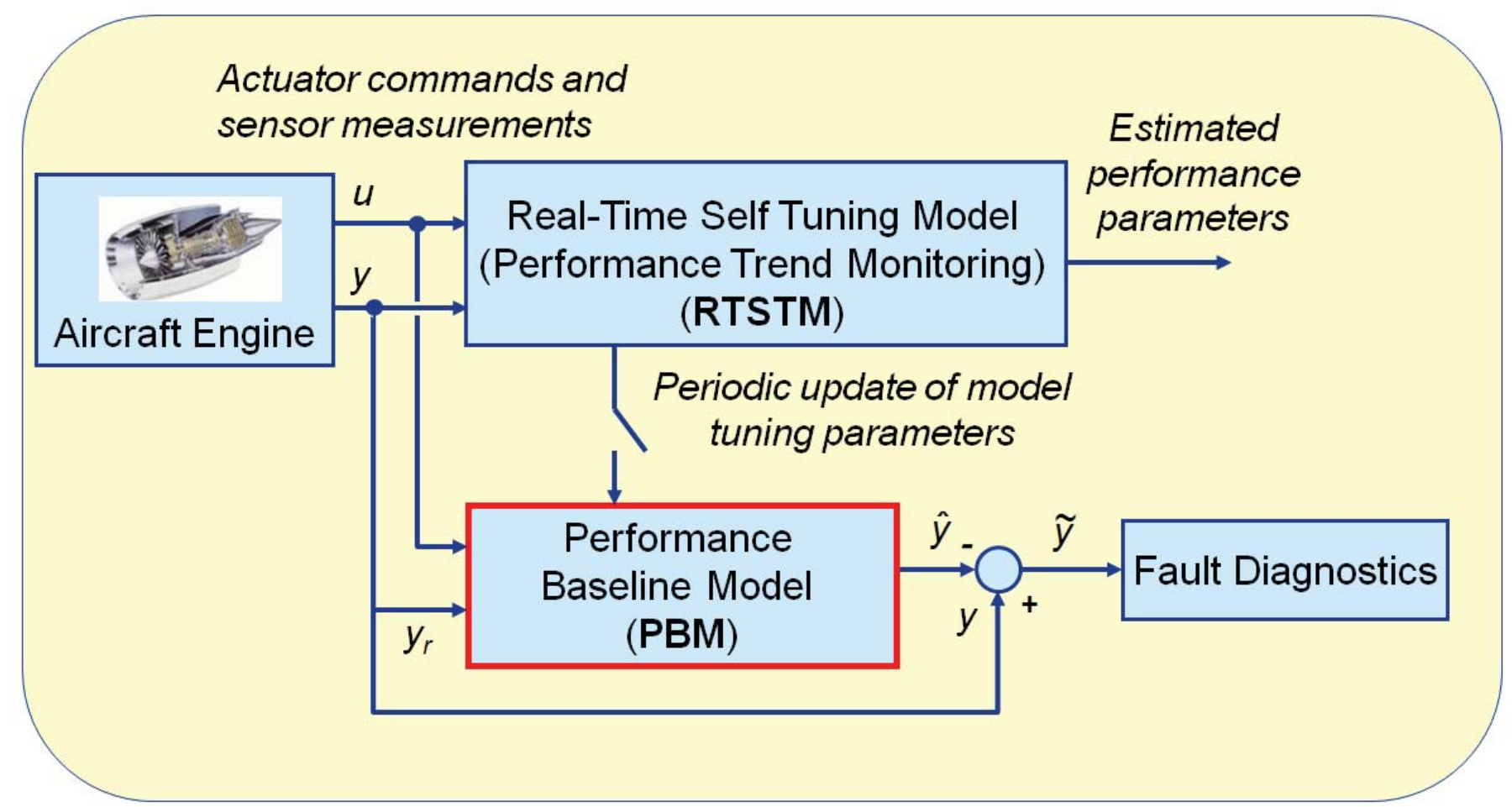

- Piecewise linear state space model design, open-loop with inputs:

- Actuator commands, $u$.

- Power reference parameter, $y_{r}$, which is used to improve model-to-engine tracking capability.

- Periodic model tuning parameter updates from RTSTM to account for gradual degradation effects.

- PBM provides a baseline of recent engine performance 


\section{Fault Diagnostics}

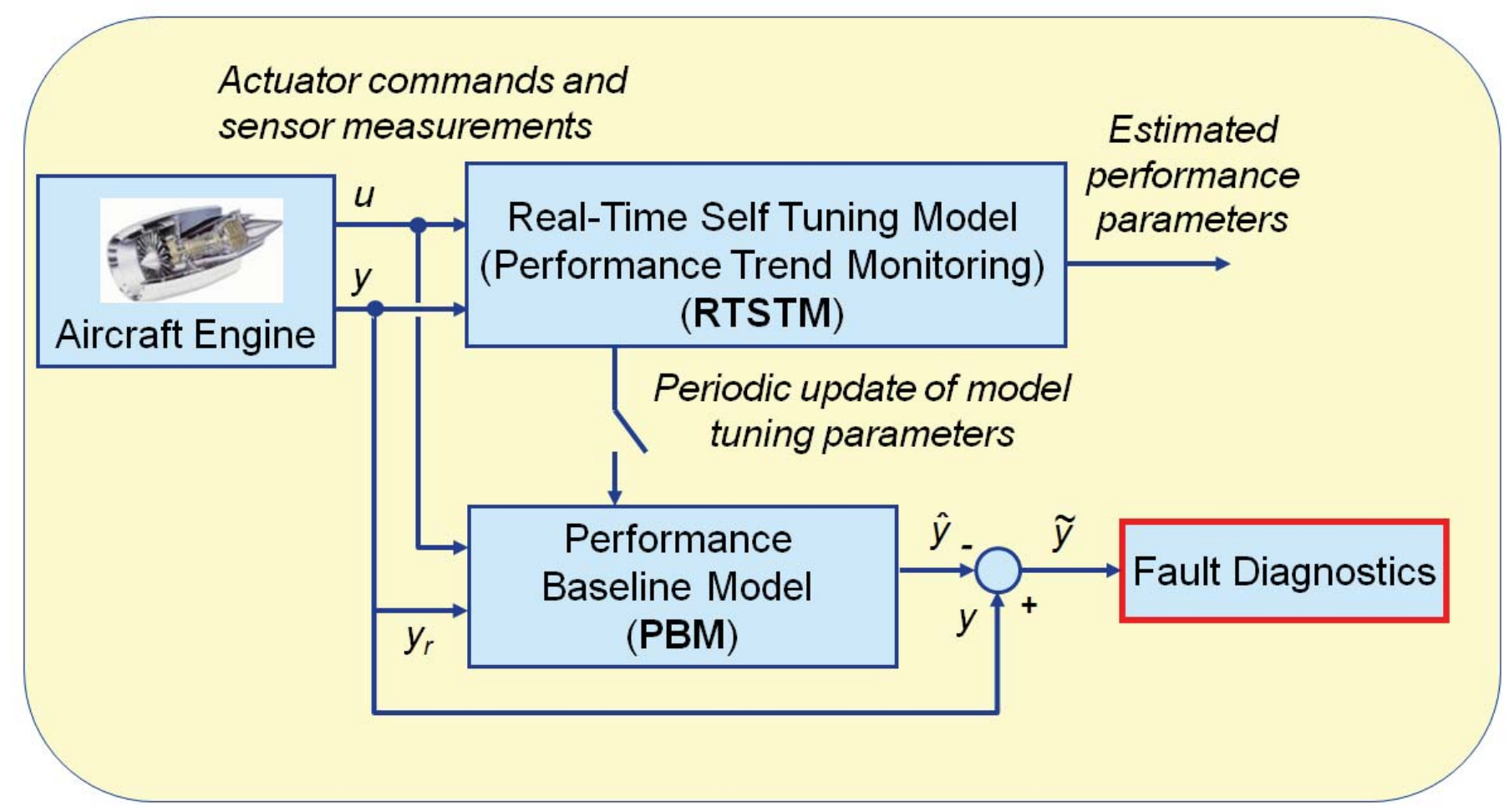

- Monitors residuals between sensed engine outputs and PBM estimated outputs

- Fault detection is performed by calculating and monitoring a weighted sum of squared residuals (WSSR) signal. WSSR $=\widetilde{y}^{\top} R^{-1} \tilde{y}$

- Upon fault detection, fault classification is performed by identifying the candidate fault signature that most closely matched the observed residual in a weighted least squares sense. WSSEE $E_{j}=\left(\widetilde{y}-\hat{\tilde{y}}_{j}\right)^{T} R^{-1}\left(\widetilde{y}-\hat{\tilde{y}}_{j}\right)$ 


\section{Fault Diagnostics}

Vantage

Partners,LLC
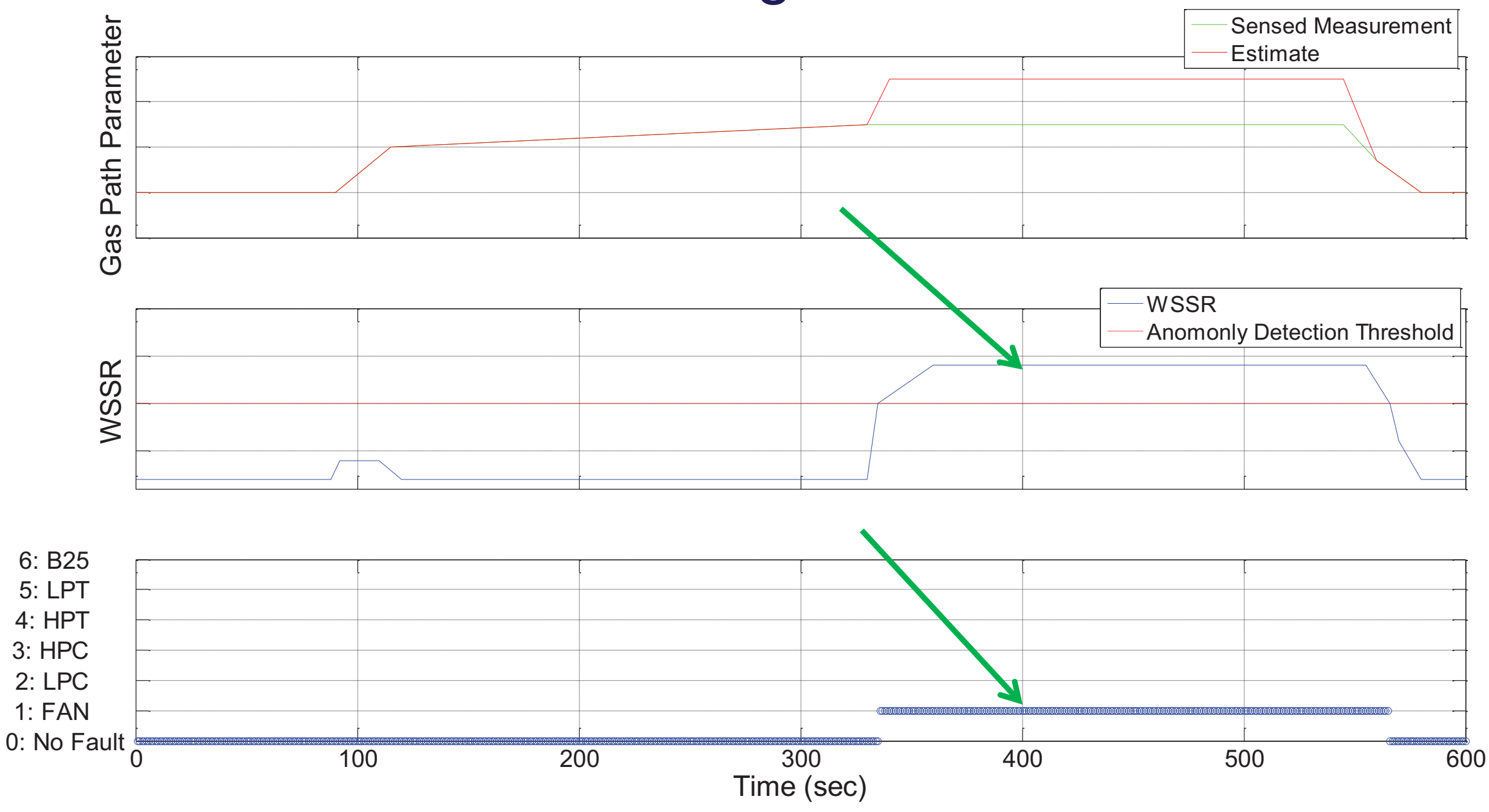

At each time sample a new WSSR and WSSEE are calculated 


\section{Fault Diagnostics}

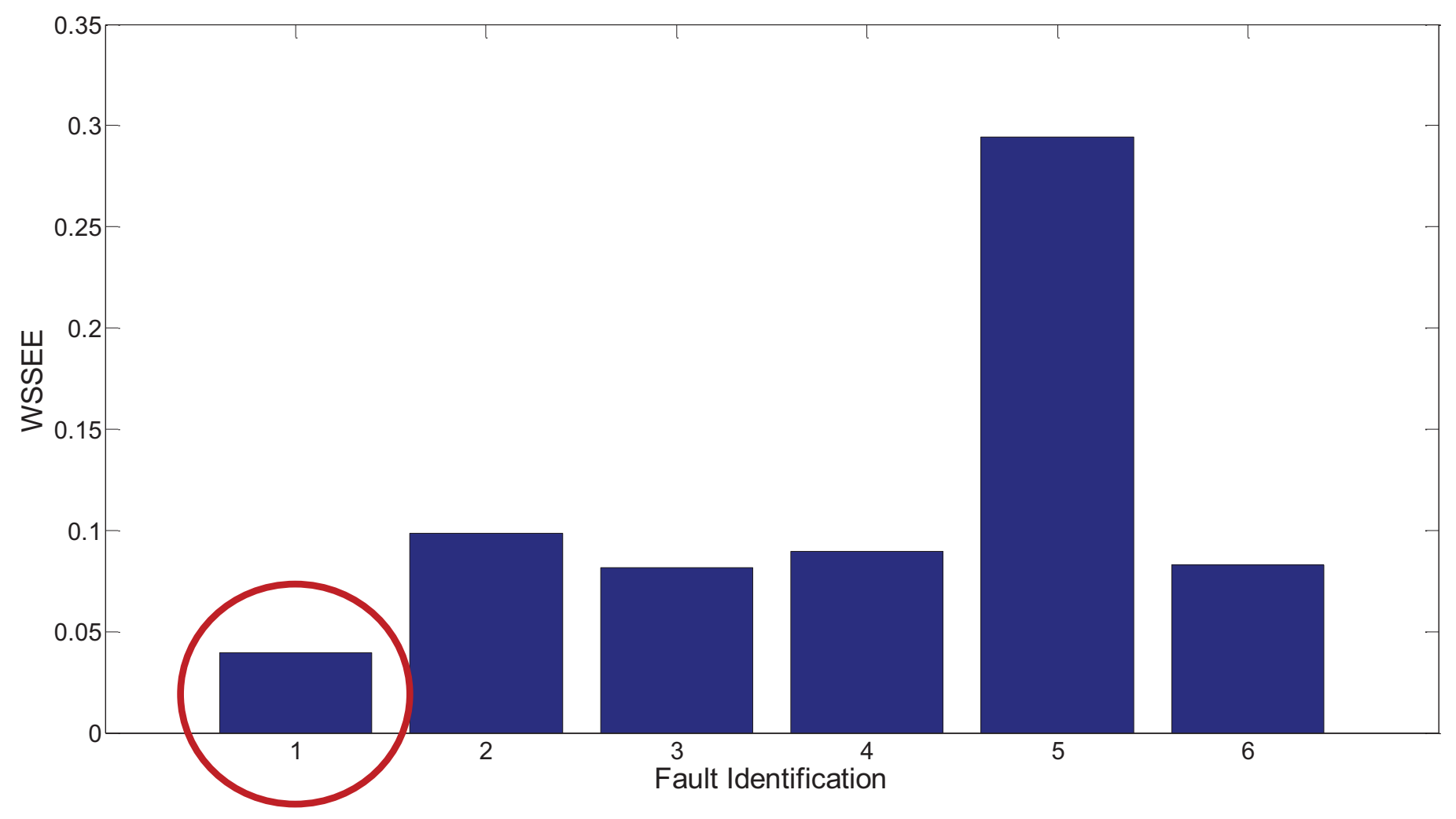

The smallest WSSEE value is classified as the fault type. 
Application Example: Analysis of Vehicle Integrated Partners,uc Propulsion Research (VIPR) Engine Test Data

- VIPR is a series of ground-based, on-wing engine tests to mature engine health management sensors and algorithms

- Ongoing at NASA Armstrong / Edwards Air Force Base

- Partners include NASA, US Air Force, Pratt \& Whitney, and others

- Test vehicle:

- Boeing C-17 Globemaster III

- Equipped with Pratt \& Whitney F117 highbypass turbofan engines

- VIPR ground tests include:

- A series of nominal and seeded faulted engine test cases

$\circ$ Faults include station 2.5 bleed valve and $14^{\text {th }}$ stage bleed valve faults

- Data collected over a range of engine power settings including steady-state and transient operating conditions

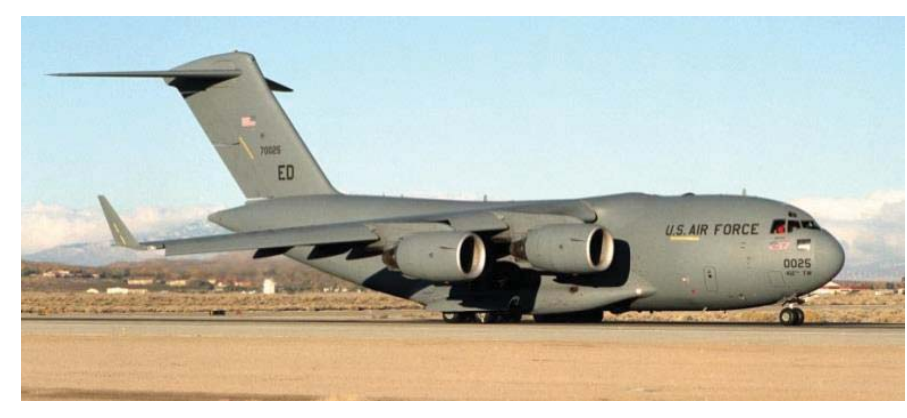

Boeing C-17 Globemaster III

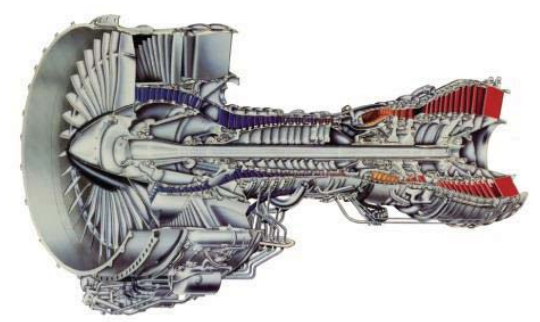

Pratt \& Whitney F117 Turbofan Engine 


\section{Model-Based Gas Path Diagnostic Architecture}

- Architecture Designed Based on NASA C-MAPSS40k Engine Model

Gas Path Sensor Measurements

\begin{tabular}{cl}
\hline \hline Symbol & Description \\
\hline N1 & fan speed \\
N2 & core speed \\
P25 & low pressure compressor exit total pressure \\
T25 & low pressure compressor exit total temperature \\
Ps3 & high pressure compressor exit static pressure \\
T35 & high pressure compressor exit total temperature \\
P5 & low pressure turbine exit total pressure \\
T5 & low pressure turbine exit total temperature \\
\hline \hline
\end{tabular}

Actuator Commands

\begin{tabular}{cl}
\hline Symbol & Description \\
\hline Wf & fuel flow \\
VSV & variable stator vanes \\
BLD25 & station 2.5 bleed valve
\end{tabular}

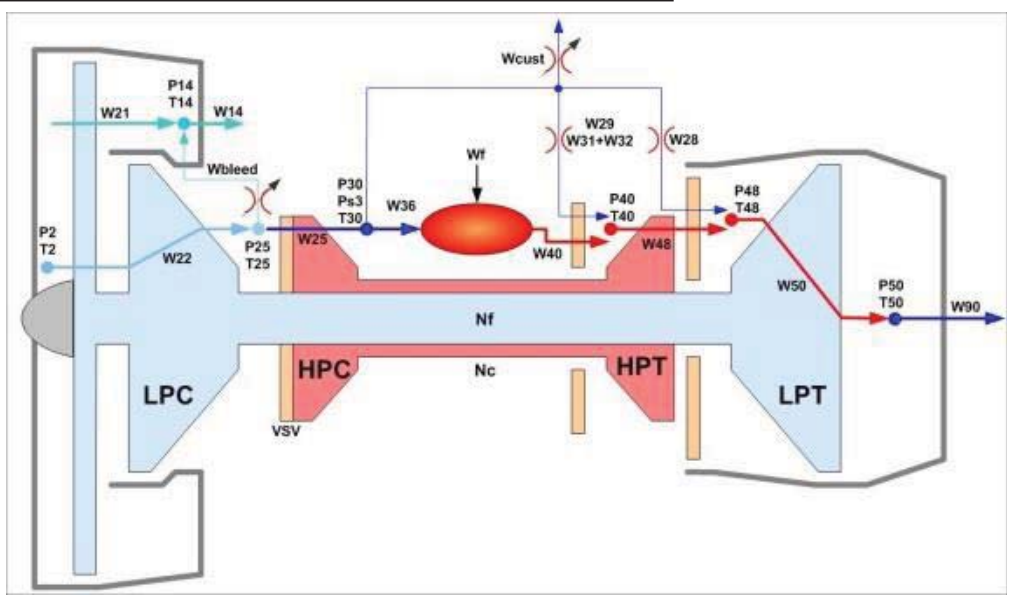

Commercial Modular Aero-Propulsion System Simulation 40k (C-MAPSS40k) 


\section{Model-Based Gas Path Diagnostic Architecture}

- Architecture Designed Based on NASA C-MAPSS40k Engine Model

\section{PBM Estimated Parameters}

Description

6 state variables ( 1 rotor speeds, 5 metal temperatures)

8 engine sensors ( 2 rotor speeds, 3 pressure and 3 temperature)

6 engine performance deterioration tuning parameters

\section{RTSTM Kalman Filter Estimated Parameters}

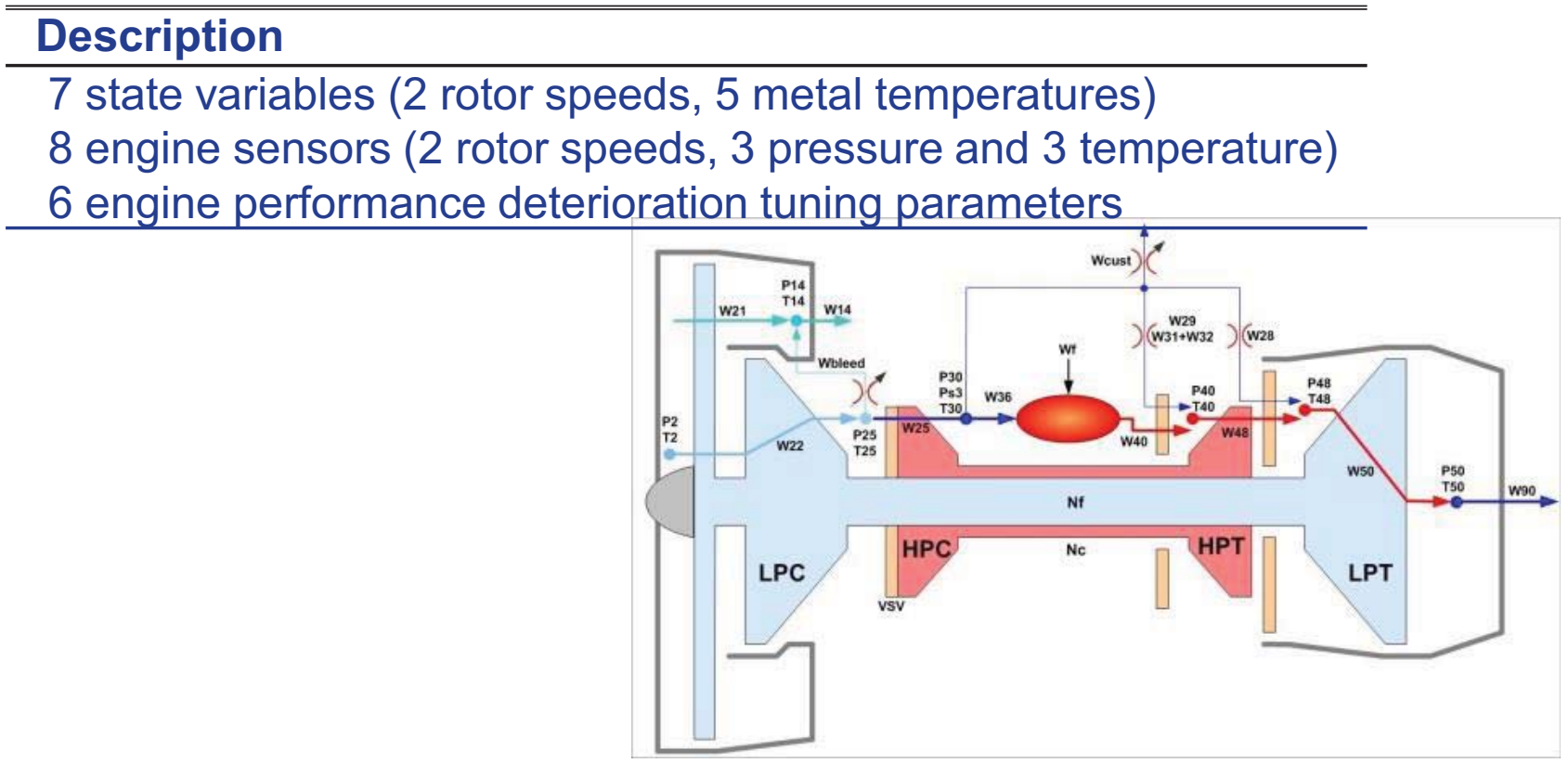

Commercial Modular Aero-Propulsion

System Simulation 40k (C-MAPSS40k) 


\section{Engine Sensors and Commands Considered in this Study}

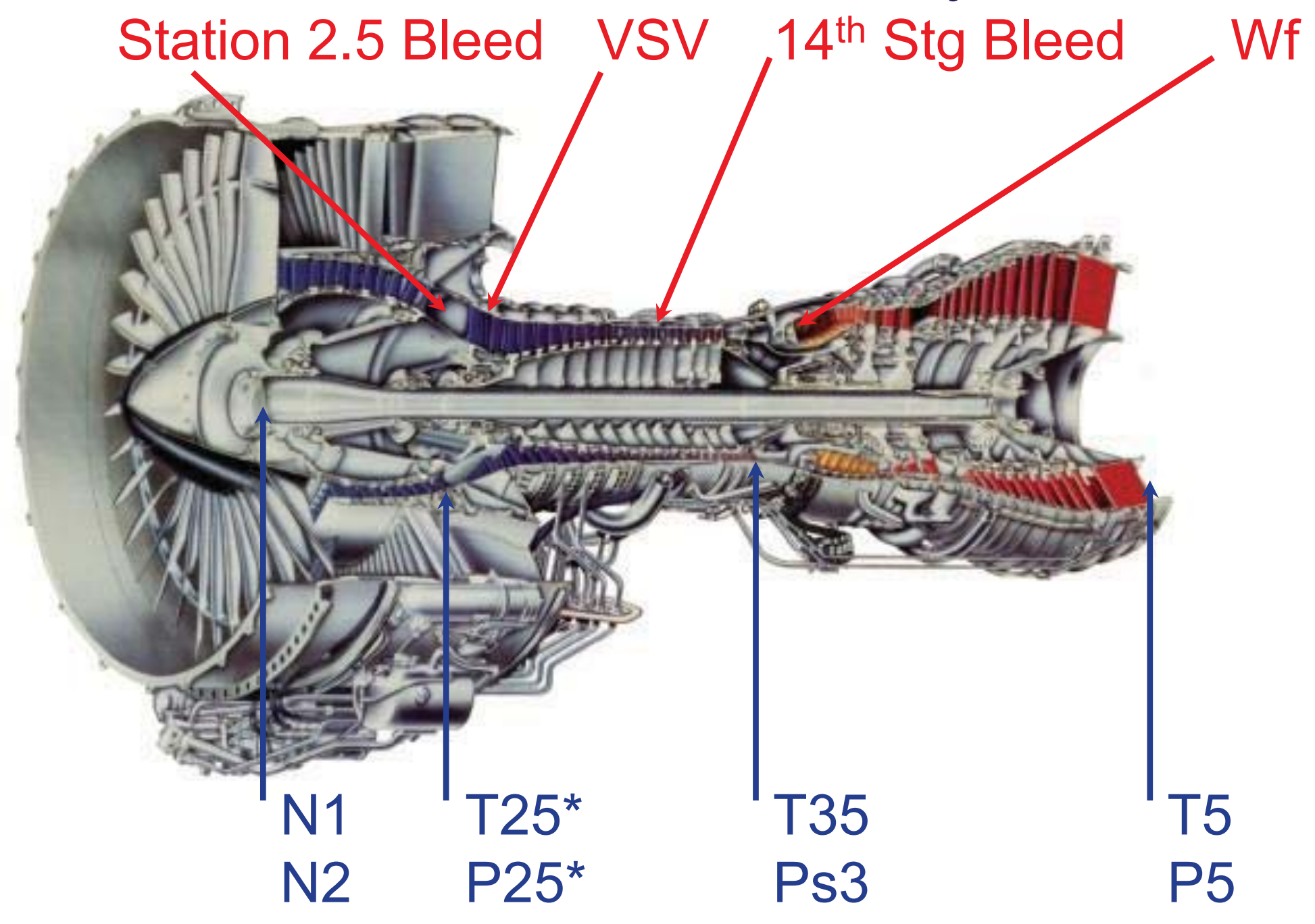

*Sensors unique to VIPR II tests 


\section{Fault Types}

\begin{tabular}{|c|c|}
\hline Fault Index & Fault Types \\
\hline $\mathbf{1}$ & Fan \\
\hline $\mathbf{2}$ & Low Pressure Compressor \\
\hline $\mathbf{3}$ & High Pressure Compressor \\
\hline $\mathbf{4}$ & High Pressure Turbine \\
\hline $\mathbf{5}$ & Low Pressure Turbine \\
\hline $\mathbf{6}$ & Station 2.5 Bleed Valve \\
\hline $\mathbf{7}$ & Variable Stator Vane \\
\hline $\mathbf{8}$ & 14 \\
\hline
\end{tabular}




\section{VIPR I Baseline Results}
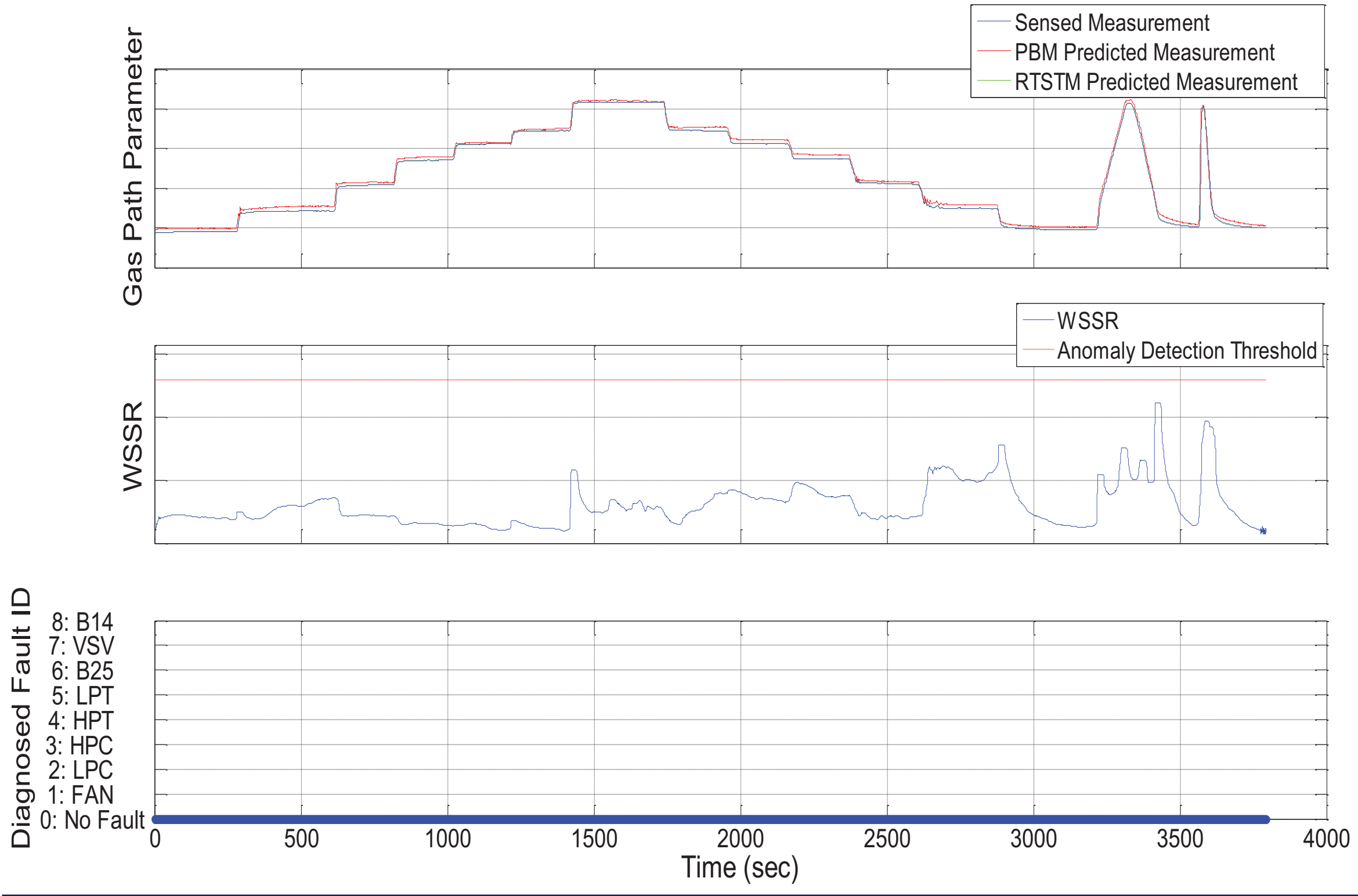


\section{VIPR I Station 2.5 Bleed Valve Fault Results}
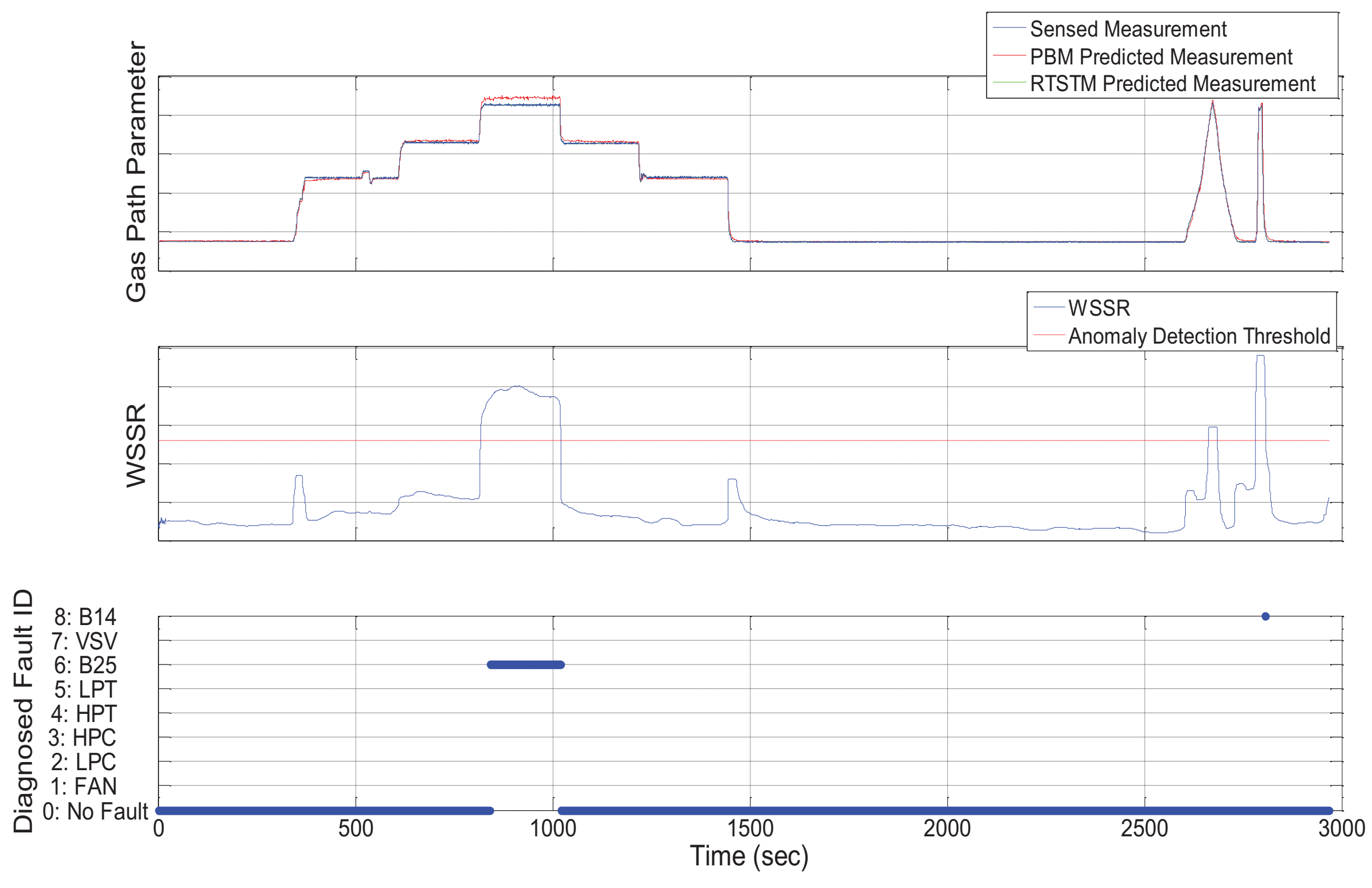
VIPR II Station 2.5 Bleed Valve Fault Results

$\underset{\substack{\text { Partingersuc } \\ \text { Pac }}}{ }$
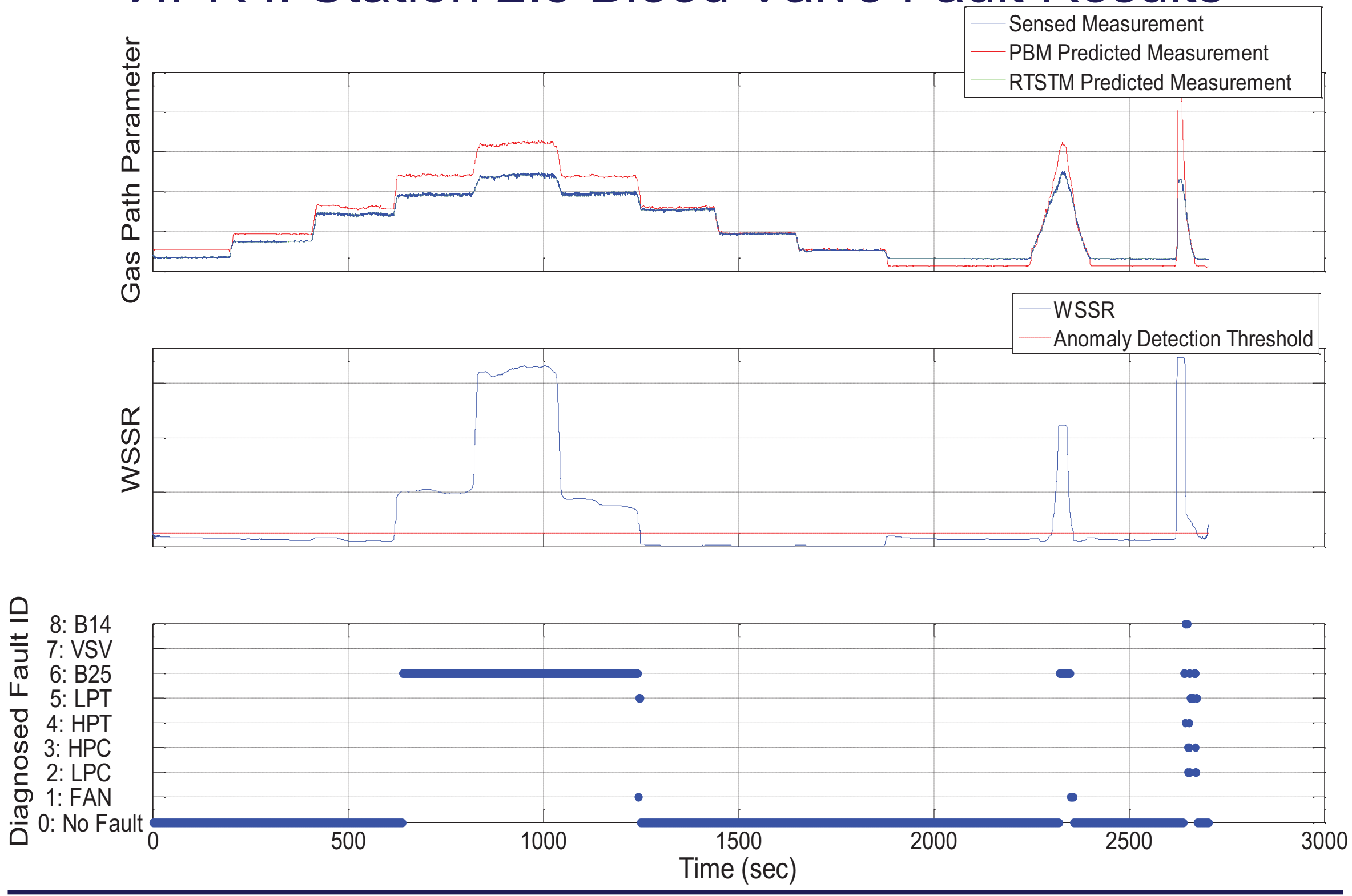


\section{VIPR I 14 ${ }^{\text {th }}$ Stage Bleed Valve Fault Results}

\section{Vantage \\ Partners, LLC}
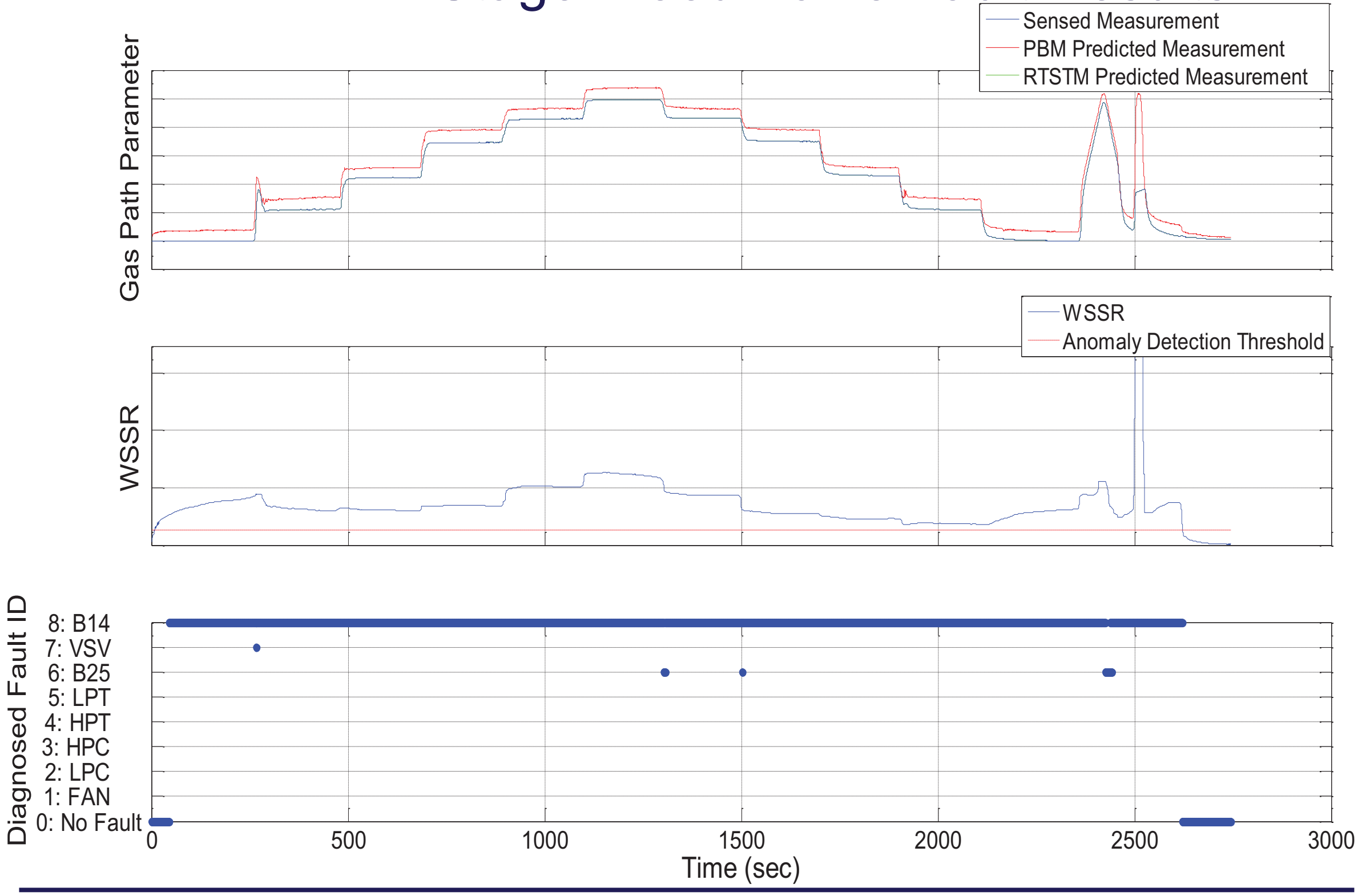


\section{VIPR II 14 $4^{\text {th }}$ Stage Bleed Valve Fault Results}
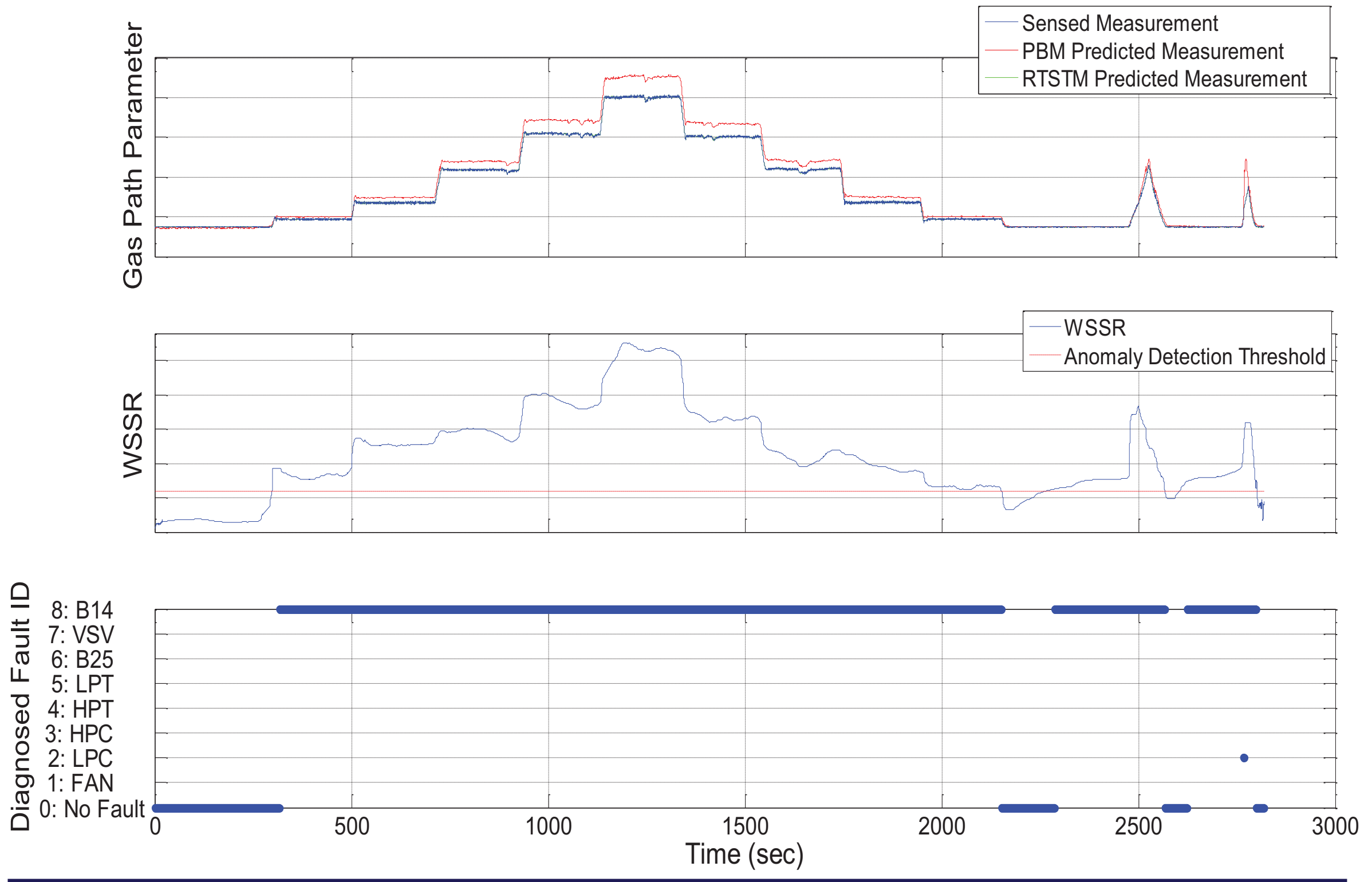


\section{Conclusion}

- Architecture was found to provide reliable steadystate fault detection and isolation

- Addition of station 2.5 sensor provided fault detection at lower power settings

- Future work will include improved matching of model to engine dynamics

- The architecture's ability to estimate deteriorated engine performance will be evaluated during the follow on VIPR III test 


\section{Acknowledgments}

Research conducted under the Vehicle Systems Safety Technologies Project of NASA's Aviation Safety Program 


\section{Backup Slides}

Vantage

Partners, LLC 


\section{Model-Based Gas Path Diagnostic Architecture Partners,uc Enhancements}

- Model-based gas path diagnostic architecture designed based on NASA C-MAPSS40k model.

- Model updates were necessary due to notable mismatch between F117 engine and C-MAPSS40k model:

$\circ$ Re-trimmed piecewise linear model to match F117 engine performance

- Updated model thermocouple dynamics

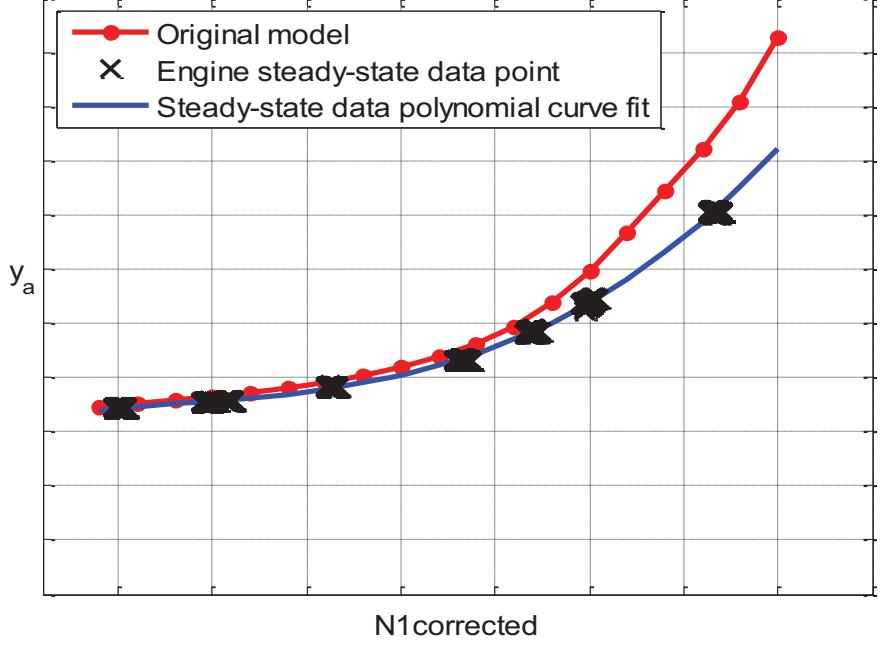

Original model and polynomial curve fit through acquired steady-state data (parameter $y_{a}$ )

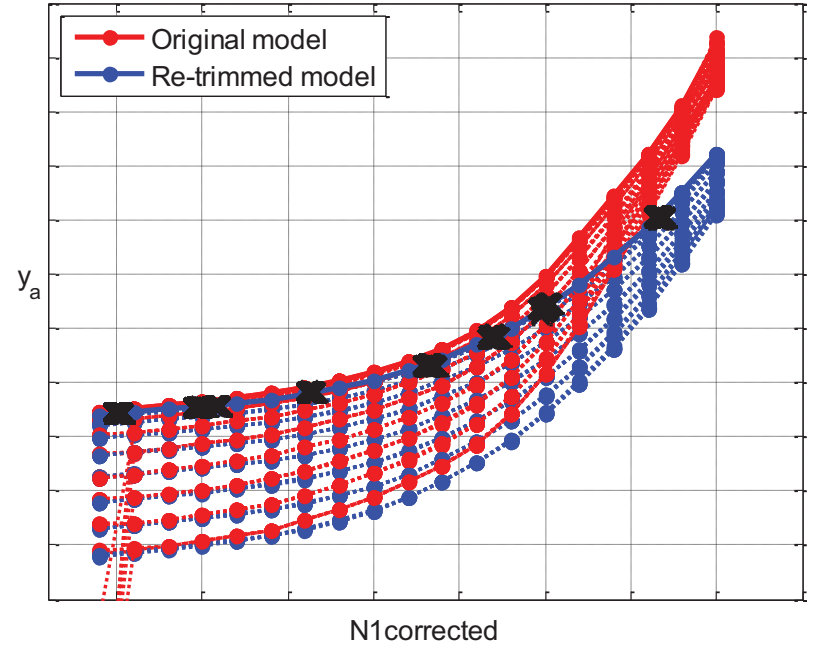

Original and re-trimmed PWLM (parameter $y_{a}$ ) 


\section{Equations}

Measurement residuals:

Weighted sum of squared residuals:

Theoretical sensor residual:

Fault influence matrix:

Estimated fault magnitude:

Estimated sensor residual:

Weighted sum of squared estimated error: $\tilde{y}=y-\hat{y}$

$W S S R=\widetilde{y}^{T} R^{-1} \tilde{y}$

$\tilde{y}=H m$

$H_{i, j}=\left[\frac{\tilde{y}_{i}}{m_{j}}\right]$

$\hat{m}_{j}=\left(H_{j}^{T} R^{-1} H_{j}\right)^{-1} H_{j}^{T} R^{-1} \tilde{y}$

$\hat{\tilde{y}}_{j}=H_{j} \hat{m}_{j}$

$\operatorname{WSSEE}_{j}=\left(\widetilde{y}-\hat{\tilde{y}}_{j}\right)^{T} R^{-1}\left(\widetilde{y}-\hat{\tilde{y}}_{j}\right)$ 


\section{VIPR II Baseline Results}
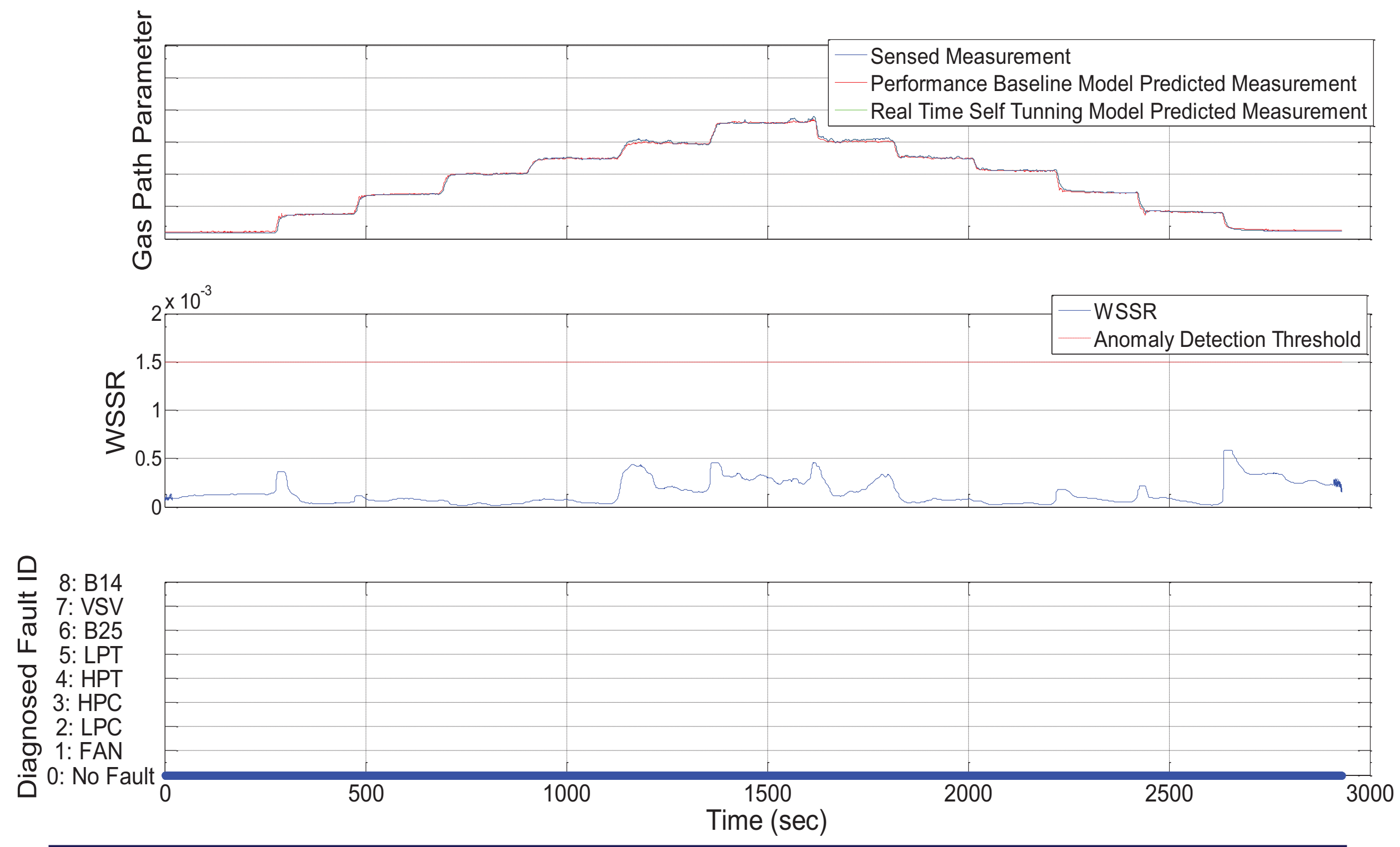